\title{
Analysis of the generation of amplitude-squeezed light with Gaussian-beam degenerate optical parametric amplifiers
}

\author{
Kahraman G. Köprülü and Orhan Aytür \\ Department of Electrical and Electronics Engineering, Bilkent University, TR-06533 Bilkent, Ankara, Turkey
}

Received August 16, 2000; revised manuscript received January 16, 2001

\begin{abstract}
We investigate the generation of amplitude-squeezed states with degenerate optical parametric amplifiers that are pumped by focused Gaussian beams. We present a model that facilitates the calculation of the squeezing level for an experimentally realistic configuration in which there is a Gaussian input signal beam that has the same confocal parameter and waist location as the Gaussian pump beam, with no restriction on the interaction length-to-confocal parameter ratio. We show that the 3-dB squeezing limit that was thought to be imposed by the Gaussian pump profile can be exceeded in the (previously uninvestigated) tight-focusing regime. We find the maximum possible amplitude squeezing in this regime to be $4.65 \mathrm{~dB}$. However, it is possible to increase the squeezing level further by spatially filtering the tails of the output signal beam, resulting in squeezing levels in excess of $10 \mathrm{~dB}$. (c) 2001 Optical Society of America
\end{abstract}

OCIS codes: $270.0270,270.6570,190.0190,190.4970$.

\section{INTRODUCTION}

A light field that exhibits a sub-Poissonian photonnumber distribution, in which the photon number's variance is less than its mean, is said to be in an amplitudesqueezed state. ${ }^{1}$ As a result of their reduced quantum noise compared with that of coherent states, these light states are attractive for a multitude of applications. So far a variety of methods have been used to generate amplitude-squeezed light. ${ }^{2-30}$ One commonly used method is phase-sensitive deamplification of a coherentstate light beam by use of a degenerate optical parametric amplifier (DOPA). ${ }^{25-30}$ In this paper we analyze the generation of amplitude-squeezed light with Gaussian-beam DOPAs, concentrating on the previously uninvestigated tight-focusing regime.

One of the earliest experiments on amplitude-squeezed light entails the demonstration of the sub-Poissonian nature of resonance fluorescence. ${ }^{2}$ After this, the generation of ultraviolet sub-Poissonian light from $\mathrm{Hg}$ vapor by inelastic collisions with a space-charge-limited electron beam was reported. ${ }^{3}$ These early experiments were followed by many others that employed a variety of methods. ${ }^{4-30}$ One important method for generating amplitude-squeezed light is based on semiconductor lasers; the sub-Poissonian characteristics of the electrical driving source are passed on to the generated laser beam by virtue of the high-gain medium. ${ }^{31}$ In an early experiment in which this concept was used, a $6-\mathrm{dB}$ reduction in amplitude noise from the quantum limit was observed at the output of a semiconductor laser that was stabilized by negative feedback ${ }^{4}$; however, this field could not be extracted from the feedback loop. Open-loop experiments $^{5-15}$ soon followed. However, achieving high levels of squeezing in these experiments required cooling the setup to cryogenic temperatures. ${ }^{5-10}$ The maximum amplitude squeezing observed in any room-temperature semiconductor laser experiment is less than $2 \mathrm{~dB} \cdot{ }^{11-15}$ The highest amplitude squeezing reported to date, however, was generated with a semiconductor laser that was cooled to $66 \mathrm{~K}$; there, $8.3 \mathrm{~dB}$ of squeezing was measured, with a detection efficiency of $89 \% .^{10}$

Another process that is utilized for generating amplitude-squeezed light is nondegenerate optical parametric downconversion, in which photons are created in pairs. In an experiment based on spontaneous parametric downconversion, 1.07-dB amplitude squeezing in one of the downconverted beams was achieved with negative feedback from the other downconverted beam to the pump. ${ }^{17}$ However, the resultant output beam had very low power $(\sim 60 \mathrm{pW})$. In another experiment, amplitudesqueezed light was obtained by use of the intensity correlations between the signal and idler beams of an optical parametric oscillator; the intensity fluctuations on the signal beam were reduced by a feed-forward correction mechanism that monitors the intensity of the idler beam, resulting in a $6-\mathrm{mW}$ output beam with a squeezing level of $1.2 \mathrm{~dB}{ }^{18}$ However, the feed-forward mechanism, and hence the squeezing, was narrow band. Kim and $\mathrm{Kumar}^{32}$ proposed a similar configuration that uses the intensity correlated twin beams from a nondegenerate optical parametric amplifier. That analysis showed that amplitude squeezing in such a case is limited only by the pumping strength.

Second-harmonic generation is also a common method of generating amplitude-squeezed light. Amplitude squeezing of the fundamental ${ }^{19,20}$ and the second-harmonic ${ }^{21}$ modes has been demonstrated in doubly resonant frequency doublers. In the research reported in Ref. 20, 3-dB squeezing of the $3.2-\mathrm{mW}$ fundamental beam was achieved by use of a monolithic magnesium oxide-doped lithium niobate $\left(\mathrm{MgO}: \mathrm{LiNbO}_{3}\right)$ doubly resonant cavity that was pumped by a Nd:YAG la- 
ser. The same configuration resulted in a $100-\mu \mathrm{W}$ second-harmonic beam with $2.2 \mathrm{~dB}$ of amplitude squeezing. ${ }^{21}$ However, both experiments required active frequency stabilization, which introduced considerable experimental complexity. A singly resonant frequency doubler was reported to generate $1.55 \mathrm{~dB}$ of squeezing in the second-harmonic mode in a monolithic $\mathrm{MgO}: \mathrm{LiNbO}_{3}$ cavity that was pumped by a Nd:YAG laser. ${ }^{22}$ In another experiment, a singly resonant potassium niobate $\left(\mathrm{KNbO}_{3}\right)$ doubler that was pumped by a Ti:sapphire laser resulted in 2.4-dB amplitude squeezing in the secondharmonic beam. ${ }^{23}$ Traveling-wave second-harmonic generation has also been used for generating amplitudesqueezed light. In one experiment, both the fundamental and the second-harmonic beams were squeezed, by 0.8 and $0.35 \mathrm{~dB}$, respectively, during second-harmonic generation in a $\mathrm{LiNbO}_{3}$ waveguide. ${ }^{24}$

DOPAs play an important role in the generation of amplitude-squeezed light. ${ }^{25-30}$ A DOPA is a phasesensitive light amplifier, whose gain depends on the optical phase difference between the pump and the input signal fields. When the input signal is in a coherent state with a sufficiently large number of photons, the deamplified output signal is in an amplitude-squeezed state. Degeneracy between the signal and idler photons necessitates that the pump frequency be exactly twice the signal frequency. In practice this is achieved by use of the second harmonic of a laser as the pump beam and of the fundamental beam as the signal input. ${ }^{25-27}$ Alternatively, the pump beam can be generated in the DOPA crystal simultaneously with parametric amplification, provided that the crystal is type II phase matched. ${ }^{28,33}$ An experiment in which a DOPA was placed inside a standing-wave cavity that is resonant for the signal field was reported to achieve 4.3-dB amplitude squeezing. ${ }^{29}$

Single-pass traveling-wave DOPAs seem to be advantageous for generating amplitude-squeezed light because of their relative simplicity, room-temperature operation, and broadband quantum noise reduction. However, amplitude squeezing greater than $0.7 \mathrm{~dB}$ had not been achieved with a single-pass DOPA because of phase distortions in the signal beam owing to the nonuniform intensity profile of the pump beam, an effect known as gaininduced diffraction (GID). ${ }^{27}$

In this paper we present an accurate and detailed analysis of generation of amplitude-squeezed light with single-pass Gaussian-beam DOPAs. In a typical experiment, the Gaussian-beam nature of the pump and the signal fields together with GID effects result in variations in the degree of amplitude squeezing in the transverse plane. A previous model brought out the importance of these effects and concluded that amplitude squeezing with a Gaussian-beam DOPA is limited to $3 \mathrm{~dB} .^{27}$ According to that model, amplitude squeezing is maximized in the limit when the length of the DOPA crystal is infinitesimally small (diffraction effects are nonexistent) and the pump power is infinitely large. This 3-dB limit is further reduced in realistic cases when the DOPA crystal has finite length and the pump beam has finite power. However, that model is valid only in the weak-focusing regime and cannot be used to calculate squeezing levels when the length of the DOPA crystal is comparable with the confo- cal parameter of the pump beam. In addition, this model contains an incorrect assumption that the maximum amplitude squeezing occurs at a pump-signal phase difference of $-\pi / 2$, the value predicted by plane-wave theory. Using our model, we show that squeezing levels greater than $3 \mathrm{~dB}$ can be obtained in the tight-focusing regime with relatively low pump power. Furthermore, the pump-signal phase difference that maximizes squeezing is significantly different from $-\pi / 2$.

The Gaussian-beam DOPA model presented in this paper facilitates accurate calculation of amplitude squeezing for all pump-signal phase differences and in all focusing regimes, provided that the parametric interaction can be considered to be perfectly phase-matched. This analysis is based on our previous study that is described in detail in Ref. 34. In Section 2 we outline our formulation for calculating amplitude squeezing in a Gaussian-beam DOPA and in Section 3 present our main results. In Section 4 we investigate ways in which amplitude squeezing may be increased by use of linear spatial filtering at the output of the DOPA.

\section{AMPLITUDE SQUEEZING WITH A DOPA}

In this section we first summarize the plane-wave theory of generation of amplitude-squeezed light with DOPAs and then present the Gaussian-beam formulation. In this analysis we assume that there is perfect phase matching, no walk-off between the pump and the signal beams, and no pump depletion. We note that it is possible to satisfy these assumptions in a carefully designed experiment.

\section{A. Plane-Wave Theory}

In the plane-wave theory of DOPAs, the amplitude $A_{s}$ of a monochromatic signal field,

$$
E_{s}(z, t)=1 / 2 A_{s}(z) \exp \left[i\left(\omega t-k_{s} z\right)\right]+\text { c.c. }
$$

is governed by

$$
\frac{\mathrm{d} A_{s}(z)}{\mathrm{d} z}=-i \kappa A_{p} A_{s}{ }^{*}(z)
$$

under the slowly varying envelope approximation, where c.c. denotes the complex conjugate of the first term, $A_{p}$ $=\left|A_{p}\right| \exp \left(i \phi_{p}\right)$ is the field amplitude of the pump at an optical frequency $2 \omega, \kappa=\omega d_{e} / n_{s} c$ is the nonlinear coupling constant, $d_{e}$ is the effective nonlinear coefficient, and $n_{s}$ is the refractive index. Because the interaction is phase matched, $k_{p}=2 k_{s}$, and, because the pump is assumed to be undepleted, $A_{p}$ is not a function of $z$. The solution of Eq. (2) for a crystal of length $l$ that is centered at $z=0$ is $^{35}$

$$
A_{s}(l / 2)=\mu A_{s}(-l / 2)-i \exp \left(i \phi_{p}\right) \nu A_{s}^{*}(-l / 2),
$$

where

$$
\begin{gathered}
\mu=\cosh \left(\kappa l\left|A_{p}\right|\right), \\
\nu=\sinh \left(\kappa l\left|A_{p}\right|\right) .
\end{gathered}
$$

The gain of the DOPA is 


$$
g(\theta)=\frac{\left|A_{s}(l / 2)\right|^{2}}{\left|A_{s}(-l / 2)\right|^{2}}=|\mu-i \exp (i \theta) \nu|^{2},
$$

where $A_{s}(-l / 2)=\left|A_{s}(-l / 2)\right| \exp \left(i \phi_{s}\right)$ and $\theta=\phi_{p}-2 \phi_{s}$ is the phase difference between the pump and the signal fields at the input.

Quantizing Eq. (3) results in ${ }^{35}$

$$
\hat{b}=\mu \hat{a}-i \exp \left(i \phi_{p}\right) \nu \hat{a}^{\dagger},
$$

where $\hat{a}$ and $\hat{b}$ are the annihilation operators associated with the signal field at the input and at the output, respectively. In an experimental configuration the input field is typically in a coherent state $|\alpha\rangle$, where $\hat{a}|\alpha\rangle$ $=\alpha|\alpha\rangle$ and $\alpha=K\left|A_{s}(-l / 2)\right| \exp \left(i \phi_{s}\right)$, where $K$ is a real normalization constant. The Fano factor at the output is

$$
F=\frac{\left\langle\Delta \hat{n}^{2}\right\rangle}{\langle\hat{n}\rangle},
$$

where $\hat{n}=\hat{b}^{\dagger} \hat{b}$ is the number operator associated with the output signal. Using Eq. (7), we find the average photon number and the variance at the output to be

$$
\begin{aligned}
\langle\hat{n}\rangle= & |\mu-i \exp (i \theta) \nu|^{2}|\alpha|^{2}+|\nu|^{2}, \\
\left\langle\Delta \hat{n}^{2}\right\rangle= & \left\{|\mu-i \exp (i \theta) \nu|^{4}\right. \\
& \left.+4 \operatorname{Im}^{2}[-i \exp (i \theta) \mu \nu]\right\}|\alpha|^{2} \\
& +2|\mu|^{2}|\nu|^{2},
\end{aligned}
$$

respectively. When the average photon number of the signal is sufficiently large, we have $|\alpha|^{2} \gg|\mu|^{2}|\nu|^{2}$, and Eqs. (9) and (10) are simplified to yield a Fano factor of

$$
\begin{aligned}
F(\theta) & =|\mu-i \exp (i \theta) \nu|^{2}+\frac{4 \operatorname{Im}^{2}[-i \exp (i \theta) \mu \nu]}{|\mu-i \exp (i \theta) \nu|^{2}} \\
& =g(\theta)+\frac{4 \operatorname{Im}^{2}[-i \exp (i \theta) \mu \nu]}{g(\theta)} .
\end{aligned}
$$

The output field is in an amplitude-squeezed state whenever $F(\theta)<1$. Maximum amplitude squeezing occurs at $\theta=-\pi / 2$, where $F(\theta)$ assumes its smallest value of $(\mu$ $-\nu)^{2}$. In this case the uncertainty in the photon number becomes less by a factor of $S=1 / \min \{F(\theta)\}$ than that of a coherent state that has the same number of average photons. Note that the maximum classic deamplification (1 over gain) has the same value as the maximum amplitude squeezing; i.e.,

$$
S=1 / \min \{F(\theta)\}=1 / \min \{g(\theta)\}=\exp \left(2 \kappa l\left|A_{p}\right|\right) .
$$

Equation (12) shows that amplitude squeezing increases without bound as the pump intensity increases.

\section{B. Gaussian-Beam Theories}

In this subsection we formulate a method for calculating the Fano factor at the output of a traveling-wave Gaussian-beam DOPA. This formulation facilitates the calculation of the Fano factor for a coherent Gaussian input signal beam that has the same confocal parameter as the pump. We consider a geometry in which the waists of the pump and the signal beams are located at the center of the nonlinear crystal, as is typical of most experimental configurations.
Because both the pump and the signal beams at the input have cylindrical symmetry, we express all fields as $+z$ propagating waves with transverse amplitude profiles that depend only on the radial distance $\rho=\sqrt{x^{2}+y^{2}}$ and on the propagation distance $z$. We consider a signal field described by

$$
E_{s}(\mathbf{r}, t)=1 / 2 A_{s}(\rho, z) \exp \left[i\left(\omega t-k_{s} z\right)\right]+\text { c.c. }
$$

Under the slowly varying envelope approximation, the evolution of the classic signal amplitude is described by ${ }^{27}$

$$
\frac{\partial A_{s}(\rho, z)}{\partial z}-\frac{1}{2 i k_{s}} \nabla_{\perp}^{2} A_{s}(\rho, z)=-i \kappa A_{p}(\rho, z) A_{s}^{*}(\rho, z),
$$

where $A_{p}(\rho, z)$ is the complex amplitude of the pump field and $\nabla_{\perp}{ }^{2}=\partial^{2} / \partial \rho^{2}+(1 / \rho) \partial / \partial \rho$ is the transverse Laplacian in cylindrical coordinates. In the case of a Gaussian pump beam, the pump field amplitude can be written as

$$
A_{p}(\rho, z)=\frac{A_{p 0}}{1-2 i z / z_{0}} \exp \left(\frac{-\rho^{2} / W_{0}^{2}}{1-2 i z / z_{0}}\right),
$$

where $W_{0}$ is the radius of the beam waist located at $z$ $=0, z_{0}=k_{p} W_{0}^{2}$ is the confocal parameter (twice the Rayleigh range), $k_{p}=2 k_{s}$ is the pump wave number, and $A_{p 0}=\left|A_{p 0}\right| \exp \left(i \phi_{p}\right)$ is a complex constant whose amplitude is related to pump power $P_{p}$ through $\left|A_{p 0}\right|$ $=\sqrt{8 \omega P_{p} / \pi z_{0} c^{2} \epsilon_{0}}$.

The signal field, $A_{s}(\rho, z)$, is a Gaussian beam at the input plane, whose waist location and confocal parameter would be identical to those of the pump beam in the absence of any nonlinear interaction. This field can be written as

$$
A_{s}(\rho, z)=\frac{A_{s 0}(\rho, z)}{1-2 i z / z_{0}} \exp \left(\frac{-\rho^{2} / 2 W_{0}^{2}}{1-2 i z / z_{0}}\right),
$$

where $A_{s 0}(\rho, z)$ is a function that represents the deviations of the signal field from an ideal Gaussian beam. In the absence of any nonlinear interaction, diffraction of the signal mode is due to propagation only, and $A_{s 0}$ is just a complex constant.

The transformations $z=\xi z_{0}$ and $\rho=r W_{0}$ can be used to normalize Eq. (14) as

$$
\begin{aligned}
\frac{\partial A_{s}(r, \xi)}{\partial \xi}+i \nabla_{\perp}^{2} A_{s}(r, \xi) & \\
= & -i \gamma \exp \left(i \phi_{p}\right) u_{p}(r, \xi) A_{s}^{*}(r, \xi),
\end{aligned}
$$

where $\phi_{p}$ is the phase of the pump beam, $\gamma=\kappa z_{0}\left|A_{p 0}\right|$ is a constant, and

$$
u_{p}(r, \xi)=\frac{1}{1-2 i \xi} \exp \left(\frac{-r^{2}}{1-2 i \xi}\right) .
$$

Equation (17) has to be solved with the initial condition $A_{s}\left(r, \xi=-\xi_{0}\right)$, where $\xi_{0}=l / 2 z_{0}$. As the signal field is purely Gaussian at the input plane, $A_{s 0}$ has no radial dependence there and $A_{s 0}\left(r,-\xi_{0}\right)=\left|A_{s 0}\left(-\xi_{0}\right)\right| \exp \left(i \phi_{s}\right)$. By substituting Eq. (16) into Eq. (17) it is possible to obtain an equation that governs the evolution of $A_{s 0}(\rho, z)$. The solution of this equation may be written in the form ${ }^{27}$ 


$$
A_{s 0}\left(r, \xi_{0}\right)=\mu(r) A_{s 0}\left(-\xi_{0}\right)+i \exp \left(i \phi_{p}\right) \nu(r) A_{s 0} *\left(-\xi_{0}\right) .
$$

The radial dependences of $\mu$ and $\nu$ are a consequence of the Gaussian profile of the pump beam.

Quantizing Eq. (19) results in

$$
\hat{b}(r)=\mu(r) \hat{a}-i \exp \left(i \phi_{p}\right) \nu(r) \hat{a}^{\dagger},
$$

provided that the input signal has perfect spatial coherence. The input signal is in a coherent state $|\alpha\rangle$, where $\alpha=K^{\prime} A_{s 0}\left(r,-\xi_{0}\right) G_{0}\left(r,-\xi_{0}\right)$, with

$$
G_{0}(r, \xi)=\frac{1}{1-2 i \xi} \exp \left(\frac{-r^{2} / 2}{1-2 i \xi}\right),
$$

where $K^{\prime}$ is a real normalization constant. Once $\mu(r)$ and $\nu(r)$ are calculated from the classic equation, the output annihilation operator is known, and we can calculate the Fano factor at the output by evaluating terms of powers of $l / z_{0}$. The results of this analysis were instrumental in bringing out the importance of GID effects, where the nonuniform gain distribution that is due to the transverse Gaussian pump profile results in a distortion of the signal phase fronts. As a result, it becomes impossible to maintain the same phase difference between the pump and the signal fields along the whole transverse plane. Consequently, different portions of the signal beam experience different levels of amplitude squeezing or amplitude desqueezing, depending on the exact local phase difference. The overall effect, integrated over both the axial and the transverse dimensions, results in a net amount of squeezing that is always less than $3 \mathrm{~dB} .{ }^{27}$ However, the solutions in this formulation are found only to the second order in $l / z_{0}$, and hence the validity of this model is limited to $l / z_{0} \ll 1$. Furthermore, for this model there is an important misconception that the maximum amplitude squeezing occurs when $\theta=-\pi / 2$, the plane-wave theoretical value.

$F=\frac{\int\left\{|\mu(r)-i \exp (i \theta) \nu(r)|^{4}+4 \operatorname{Im}^{2}\left[-i \exp (i \theta) \mu^{*}(r) \nu(r)\right]\right\}\left|G_{0}\left(r, \xi_{0}\right)\right|^{2} r \mathrm{~d} r}{\int|\mu(r)-i \exp (i \theta) \nu(r)|^{2}\left|G_{0}\left(r, \xi_{0}\right)\right|^{2} r \mathrm{~d} r}$.

\section{A. No Diffraction Limit}

The no-diffraction limit, at which $l / z_{0} \rightarrow 0$, provides an approximate but simple solution and illustrates some of the limitations on squeezing imposed by the Gaussian pump profile. In this case the diffraction (transverse Laplacian) term in Eq. (17) vanishes, and $\mu(r)$ and $\nu(r)$ are found $\mathrm{as}^{36}$

$$
\begin{gathered}
\mu(r)=\cosh \left[\Phi \exp \left(-r^{2}\right)\right], \\
\nu(r)=\sinh \left[\Phi \exp \left(-r^{2}\right)\right],
\end{gathered}
$$

where $\Phi=\gamma l / z_{0}=\kappa l\left|A_{p 0}\right|$. Inserting $\mu(r)$ and $\nu(r)$ into Eq. (22) yields the maximum amplitude squeezing ${ }^{27}$ :

$$
S=1 / \min \{F(\theta)\}=\frac{2[1-\exp (-2 \Phi)]}{1-\exp (-4 \Phi)} .
$$

This maximum squeezing occurs at $\theta=-\pi / 2$, as in the plane-wave theory. As the pump power increases, the squeezing level increases monotonically; in the limit as the pump power goes to infinity $(\Phi \rightarrow \infty)$, the squeezing level asymptotically reaches $3 \mathrm{~dB}$. The transverse gain variation that results from the Gaussian pump profile results in higher squeezing at the center and lower squeezing at the tails of the beam. Integration over the transverse plane results in the $3-\mathrm{dB}$ limit on the maximum obtainable amplitude squeezing.

\section{B. Weak-Focusing Limit}

The weak-focusing limit where $l / z_{0} \ll 1$ was analyzed by Li et al. ${ }^{27}$ In this analysis, an approximate solution for $A_{s}(\rho, z)$ was obtained by use of a perturbative method in

\section{General Solution}

Our model presented in this paper allows us to calculate the squeezing level for arbitrary values of $l / z_{0}$ and $\theta$. The results of our model show that it is possible to break the 3-dB limit in the tight-focusing regime by a careful choice of the initial phase difference and pump power. In our approach we solve Eq. (17) by numerical techniques. ${ }^{34}$ Here the signal mode is expressed as the sum of an infinite number of Laguerre-Gaussian beams in cylindrical coordinates $(r, \varphi, \xi)$. As the configuration at hand has cylindrical symmetry, only the cylindrically symmetric modes are taken into account. When this is done, the partial differential equation that describes the evolution of the signal field can be written as an ordinary differential equation in matrix form. ${ }^{34}$ This new form of the equation is useful not only because it is more suitable for numerical techniques but also because it provides better physical insight into the processes involved. Using this model, we determine the maximum possible amplitude squeezing that can be generated with a Gaussian-beampumped DOPA.

First we write the complex amplitude of the signal field as

$$
A_{s}(r, \xi)=\sum_{n=0}^{\infty} A_{n}(\xi) G_{n}(r, \xi),
$$

where $A_{n}$ is the complex amplitude of the $n$th cylindrically symmetric Laguerre-Gaussian mode:

$$
\begin{aligned}
G_{n}(r, \xi)= & L_{n}\left(\frac{r^{2}}{1+4 \xi^{2}}\right) \frac{1}{1-i 2 \xi} \\
& \times \exp \left(\frac{-r^{2} / 2}{1-i 2 \xi}\right) \exp \left(i 2 n \tan ^{-1} 2 \xi\right),
\end{aligned}
$$


where $L_{n}$ is the $n$ th-order Laguerre polynomial. Using this mode expansion, we represent $A_{s}$ as a vector $A$ whose elements are the coefficients $A_{n}$ of this expansion. The solution of Eq. (17) can now be written in matrix form as ${ }^{34}$

$$
A\left(\xi_{0}\right)=M A\left(-\xi_{0}\right)-i \exp \left(i \phi_{p}\right) N A^{*}\left(-\xi_{0}\right),
$$

where $M\left(\gamma, \xi_{0}\right)$ and $N\left(\gamma, \xi_{0}\right)$ are state transition matrices that relate the output to the input signal field. The elements of $M$ and $N$ are calculated numerically by finitedifference techniques; the details of these calculations can be found in Ref. 34 .

For the case at hand, the input signal consists of the fundamental mode only; i.e., $A_{s}\left(r,-\xi_{0}\right)=A_{0}\left(-\xi_{0}\right)$ $\times G_{0}\left(r,-\xi_{0}\right)$. With this equation, Eq. (26) becomes

$$
\begin{aligned}
A_{s}\left(r, \xi_{0}\right)= & G_{0}\left(r, \xi_{0}\right) \sum_{n=0}^{\infty} L_{n}\left(\frac{r^{2}}{1+4 \xi_{0}^{2}}\right) \\
& \times \exp \left(i 2 n \tan ^{-1} 2 \xi_{0}\right) M_{n 0} A_{0}\left(-\xi_{0}\right) \\
& +G_{0}\left(r, \xi_{0}\right) \sum_{n=0}^{\infty} L_{n}\left(\frac{r^{2}}{1+4 \xi_{0}^{2}}\right) \\
& \times \exp \left(i 2 n \tan ^{-1} 2 \xi_{0}\right) N_{n 0} A_{0} *\left(-\xi_{0}\right) .
\end{aligned}
$$

Once $M$ and $N$ are calculated as detailed in Ref. 34, Eqs. (16) and (19) are compared with Eq. (29) to yield $\mu(r)$ and $\nu(r)$ as

$$
\begin{aligned}
& \mu(r)=\sum_{n=0}^{\infty} L_{n}\left(\frac{r^{2}}{1+4 \xi_{0}^{2}}\right) \exp \left(i 2 n \tan ^{-1} 2 \xi_{0}\right) M_{n 0}, \\
& \nu(r)=\sum_{n=0}^{\infty} L_{n}\left(\frac{r^{2}}{1+4 \xi_{0}^{2}}\right) \exp \left(i 2 n \tan ^{-1} 2 \xi_{0}\right) N_{n 0} .
\end{aligned}
$$

We use these equations in turn to calculate the Fano factor at the output from Eq. (22).

\section{Error Analysis}

Determining the Fano factor involves two separate numerical calculations: First the elements of the matrices $M$ and $N$ are found, and then the integrals in Eq. (22) are evaluated. It is important to examine various sources of numerical error in these calculations.

The first source of error stems from the finite step size in the variable $\xi$ when finite differencing is used to calculate the elements of $M$ and $N$. This error is analyzed in detail in Ref. 34. For our calculations we chose the step size such that the errors in the amplitudes of the elements of $M$ and $N$ were less than $5 \times 10^{-4}$.

The second source of error comes from truncating the infinite summation of Eq. (26) and the matrices $M$ and $N$. Inasmuch as the input signal consists of the fundamental mode only, considering a finite number of higher-order modes at the output is sufficient to represent the signal with negligible error. In our calculations we truncate the modal expansion of Eq. (26) at 20 modes, and the matrices $M$ and $N$ at $20 \times 20$ size. It turns out that this truncated equation is sufficient to represent the signal field at the output with an error that is a few orders of magnitude less than that which is due to the finite step size. ${ }^{34}$

The third source of error is due to truncating the summations in Eqs. (30) and (31) at 20 terms. As a conse- quence, the functions $\mu(r)$ and $\nu(r)$ are accurately known only for a limited radial range $0<r<r_{\max }$. We can determine the value of $r_{\max }$ by examining the signal mode amplitudes at the output. We found that the exact value of $r_{\text {max }}$ depends on various DOPA parameters; however, it is always greater than $5 \sqrt{1+4 \xi_{0}{ }^{2}}$. Compared with the other sources of error, this one is relatively small because the fraction of the signal power that lies outside the $r=r_{\text {max }}$ circle is orders of magnitude less than the error induced by the finite step size.

The last source of error is due to the integrations in Eq. (22). These integrals are calculated numerically from trapezoidal integration. However, as the values of the function inside the integral is known for all values of $r$, the finite step size in $r$ may be chosen small enough that the error that is due to the finite step size is a few orders of magnitude less than the other errors.

In our calculations, the collective effect of all four sources of error results in an error in the Fano factor that is less than $0.3 \%(0.013 \mathrm{~dB})$.

\section{RESULTS AND DISCUSSION}

The formulation outlined in Section 2 allows us to calculate the Fano factor for arbitrary values of $\theta, \gamma$, and $l / z_{0}$. That the constant,

$$
\gamma=\kappa z_{0}\left|A_{p 0}\right|=\frac{2 d_{e}}{n c^{2}}\left(\frac{2 \omega^{3} z_{0} P_{p}}{\pi \epsilon_{0}}\right)^{1 / 2}
$$

is proportional to the effective nonlinear coefficient $d_{e}$, and the square root of the pump power $P_{p}$ $=\pi z_{0} c^{2} \epsilon_{0}\left|A_{p 0}\right|^{2} / 8 \omega$, is a measure of how strongly the DOPA is pumped. ${ }^{34}$ The ratio $l / z_{0}$ is a measure of how tightly the pump beam is focused onto the crystal.

As an example of the results of our formulation, Fig. 1 shows a contour plot of the Fano factor as a function of $\gamma$ and $\theta$ while $l / z_{0}$ is kept constant at a value of 1.5 . The minimum Fano factor of $-4.61 \mathrm{~dB}$ is marked by a cross in the figure. Note that the phase difference $\theta=\theta_{m}$ at which the minimum Fano factor (maximum amplitude squeezing) occurs is significantly different from $-\pi / 2$. At any fixed value of $\theta$, however, there is an optimal value of $\gamma$ that minimizes the Fano factor. Increasing $\gamma$ further from this optimal value increases the Fano factor (decreases squeezing) as a result of increased GID effects.

From an experimental point of view, it is more relevant to investigate the nonlinear drive, defined as

$$
D=\left(\frac{8 \omega}{\pi c^{2} \epsilon_{0}}\right)\left(\kappa^{2} l P_{p}\right)=\left(\frac{l}{z_{0}}\right) \gamma^{2},
$$

than $\gamma \cdot{ }^{34}$ In a typical experimental setting, the maximum available $D$ is limited by laser power $P_{p}$, crystal length $l$, and effective nonlinear coefficient $d_{e}$. It is usu- 


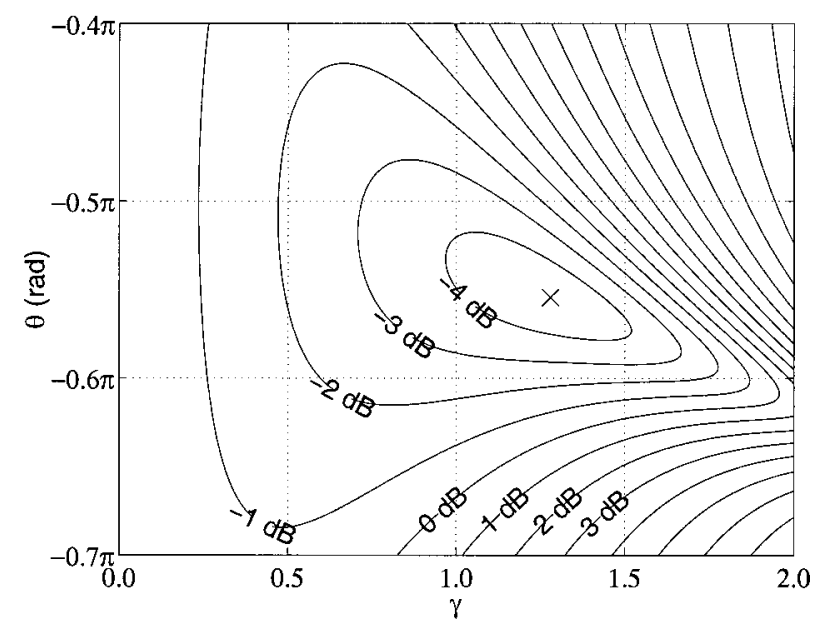

Fig. 1. Contour plot of the Fano factor as a function of $\theta$ and $\gamma$ for $l / z_{0}=1.5$. The minimum Fano factor of $-4.61 \mathrm{~dB}$ is marked $\times$.
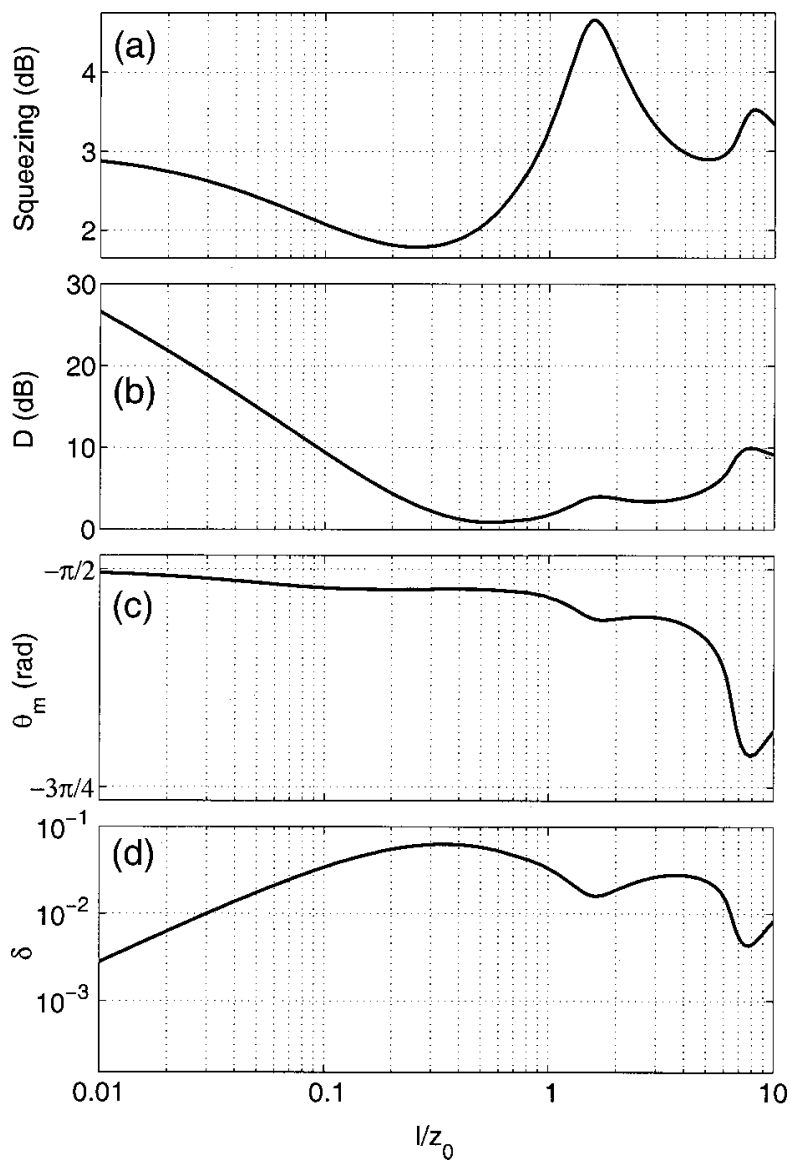

Fig. 2. (a) Maximum squeezing $S_{\max }=1 / \min \{F(\theta, \gamma)\}$, (b) required nonlinear drive $D$, (c) optimal phase difference $\theta_{m}$, and (d) normalized $10 \%(0.41-\mathrm{dB})$ phase width $\delta$, all as functions of $l / z_{0}$.

ally possible to adjust the value of $z_{0}$, and hence that of $l / z_{0}$, by changing the focused spot size of the pump and the signal beams.

Another important quantity in our calculations is the sensitivity of the Fano factor to fluctuations in the phase difference $\theta$ about its optimal point at $\theta_{m}$. In an experiment, the phase difference between the pump and the signal fluctuated in time by a certain amount, even when active stabilization was employed, resulting in a degradation of the maximum observed amplitude squeezing. ${ }^{25,27}$ We define the normalized $10 \%(0.41 \mathrm{~dB})$ phase width of the Fano factor as $\delta=\Delta \theta / 2 \pi$, where $\Delta \theta$ is the full width at $1.1 \times \min \{F(\theta)\}$. In an experiment, if the phase fluctuations became larger than $\delta$, the maximum squeezing that could be achieved began to deviate significantly from $1 / \min \{F\}$. Therefore it is desirable to operate at a large value of $\delta$.

To determine the maximum possible amplitude squeezing for a given value of $l / z_{0}$ we compute the $\theta$ and $\gamma$ values that minimize the Fano factor by using numerical optimization algorithms. Figure 2 shows the maximum possible squeezing $[S=1 / \min \{F(\theta, \gamma)\}]$ as a function of $l / z_{0}$. The optimal $\theta$ and $D$ values that maximize squeezing at each $l / z_{0}$ value are also included in the figure. Note that, for each $l / z_{0}$ value, $D$ and $\gamma$ are related through Eq. (33). The normalized phase width $\delta$ is also included in the figure.

In the weak-focusing regime where $l / z_{0} \ll 1$, the squeezing level is close to $3 \mathrm{~dB}$ and the phase difference that maximizes squeezing level $\theta_{m}$ is close to $-\pi / 2$. These values are as expected because GID effects are small in this regime. Note that the normalized phase width $\delta$ is relatively narrow (less than $10^{-2}$ for $l / z_{0}$ $<0.03$ ) and that the nonlinear drive $D$ required for maximum squeezing is quite high. In this region, decreasing $l / z_{0}$ to obtain better squeezing is useless because the required nonlinear drive increases exponentially, whereas the maximum possible squeezing approaches 3 $\mathrm{dB}$ asymptotically.

As $l / z_{0}$ is increased from zero, the maximum possible squeezing decreases to $l / z_{0}=0.25$, whereas $\theta_{m}$ deviates from $-\pi / 2$ and the required $D$ decreases. However, for $l / z_{0}>0.25$, squeezing begins to increase while the required $D$ levels off at $\sim 1 \mathrm{~dB}$. Even though $\delta$ begins to decrease from its maximum value near $l / z_{0}=0.3$, it nevertheless stays relatively high. In the range $0.9<l / z_{0}$ $<4.0$, the $3-\mathrm{dB}$ limit is clearly surpassed. In this regime, $\theta_{m}$ is significantly different from $-\pi / 2$, and $\delta$ stays greater than $\sim 10^{-2}$. The highest squeezing is $4.65 \mathrm{~dB}$ and occurs at $l / z_{0}=1.6$, where the optimal $D$ value is 4 $\mathrm{dB}$, and $\delta=1.6 \times 10^{-2}$.

It is clear that working in the $1.2<l / z_{0}<2.1$ focusing range is much more advantageous than working in the weak-focusing regime. In this tight-focusing regime it is possible to obtain amplitude-squeezing levels in excess of $4 \mathrm{~dB}$ with much lower nonlinear drive and better phase stability.

\section{HIGHER SQUEEZING WITH LINEAR SPATIAL FILTERING}

The amplitude-squeezed output signal beam exhibits a radial dependence of the squeezing level on the transverse plane, as indicated by Eqs. (20), (30), and (31). Therefore it is possible to obtain higher levels of net squeezing through linear spatial filtering of this beam, 
which we can do simply by placing a pinhole that is centered on the signal beam at the output of the DOPA. The pinhole transmits the higher-squeezed central portion of the signal beam and blocks the lower-squeezed tails. The net effect, integrated over the transverse plane, is an enhancement of overall squeezing.

It seems counterintuitive to enhance squeezing by introducing some kind of linear loss. In general, at any transverse point on the output signal beam the squeezing level is related to all the annihilation operators that correspond to all the points along the transverse plane at the input. However, the perfect spatial coherence of the input signal beam allows us to represent the input as a single annihilation operator multiplied by a Gaussian profile. Consequently it is possible to define amplitude squeezing point by point along the transverse plane at the output.

It is convenient to represent the radius of the filtering pinhole in terms of the radius of the pump beam at the output of the DOPA. For a pinhole of physical radius $\rho_{\mathrm{ph}}$, the normalized pinhole radius is defined as $R_{\mathrm{ph}}$ $=\rho_{\mathrm{ph}} / W_{p}$, where $W_{p}=W_{0} \sqrt{1+4 \xi_{0}{ }^{2}}$ is the physical radius of the pump beam at the output plane. When we define a new variable, $R=r / \sqrt{1+4 \xi_{0}^{2}}$, Eqs. (30) and (31) become

$$
\begin{aligned}
& \mu(R)=\sum_{n=0}^{\infty} L_{n}\left(R^{2}\right) \exp \left(i 2 n \tan ^{-1} 2 \xi_{0}\right) M_{n 0}, \\
& \nu(R)=\sum_{n=0}^{\infty} L_{n}\left(R^{2}\right) \exp \left(i 2 n \tan ^{-1} 2 \xi_{0}\right) N_{n 0},
\end{aligned}
$$

and we can rewrite Eq. (22) as
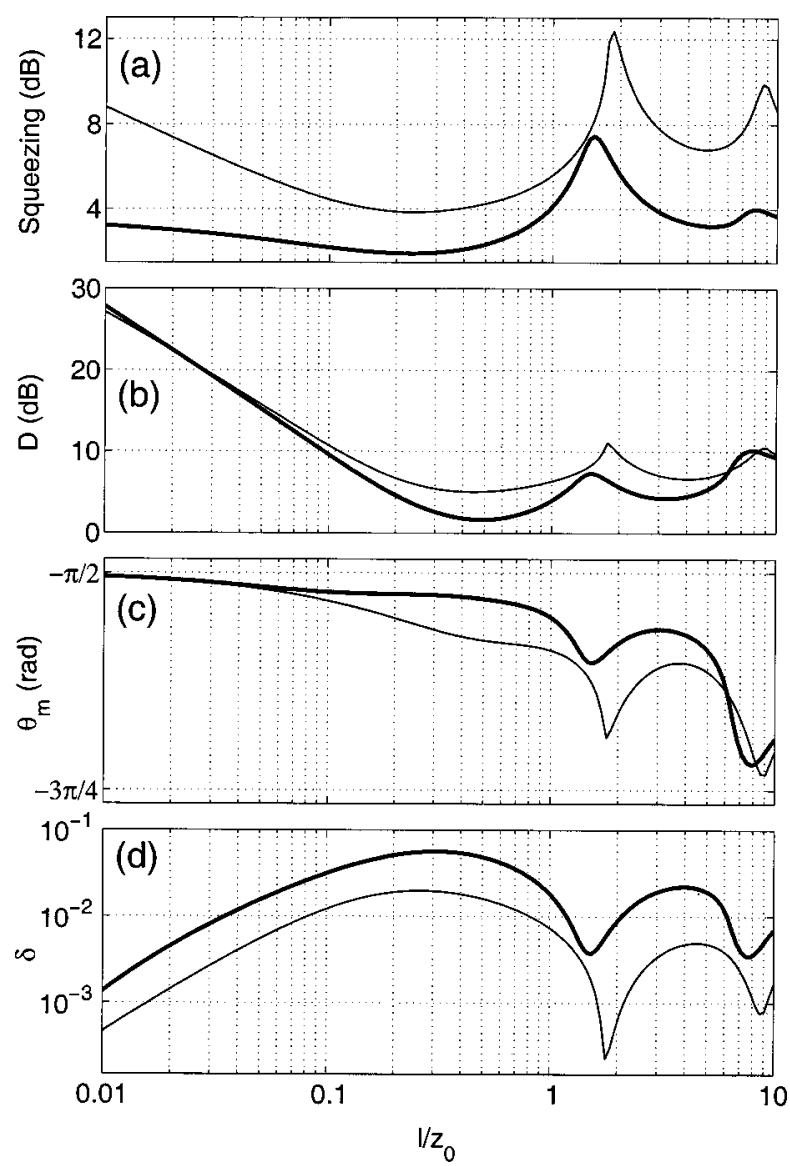

Fig. 3. (a) Maximum squeezing $S_{\max }=1 / \min \{F(\theta, \gamma)\}$, (b) required nonlinear drive $D$, (c) optimal phase difference $\theta_{m}$, and (d) normalized $10 \%(0.41-\mathrm{dB})$ phase width $\delta$, all as functions of $l / z_{0}$ for normalized pinhole radius $R_{\mathrm{ph}}=2$ (thicker curves) and $R_{\mathrm{ph}}=1$ (thinner curves).

$F=\frac{\int\left\{|\mu(R)-i \exp (i \theta) \nu(R)|^{4}+4 \operatorname{Im}^{2}\left[-i \exp (i \theta) \mu^{*}(R) \nu(R)\right]\right\} \exp \left(-R^{2}\right) R \mathrm{~d} R}{\int|\mu(R)-i \exp (i \theta) \nu(R)|^{2} \exp \left(-R^{2}\right) R \mathrm{~d} R}$

It is now straightforward to calculate the Fano factor of the filtered beam by limiting the boundaries of the integrals in Eq. (36) to the normalized pinhole radius $R_{\mathrm{ph}}$.

Figure 3 shows our results for two values, $R_{\mathrm{ph}}=1$ and $R_{\mathrm{ph}}=2$, of the normalized pinhole radius. In these calculations the Fano factor is optimized in $\gamma$ and $\theta$ after the integration boundaries are limited to $R_{\mathrm{ph}}$. Note that a dramatic improvement in squeezing is achieved as the pinhole radius is decreased. For the wider pinhole $\left(R_{\mathrm{ph}}\right.$ $=2$ ), which transmits $96 \%$ of the total signal beam power, the maximum squeezing is $7.45 \mathrm{~dB}$ at $l / z_{0}$ $=1.54$. It is surprising that only a $4 \%$ power loss results in 2.8-dB-higher squeezing. The narrower pinhole $\left(R_{\mathrm{ph}}=1\right.$ ), on the other hand, transmits $47 \%$ of the signal power and results in a maximum squeezing of $12.4 \mathrm{~dB}$ at $l / z_{0}=1.86$. Clearly, the reduced output power is not a big penalty to pay for the increase in squeezing. Simi- larly, the increase in the required nonlinear drive is within acceptable limits.

The actual price paid for increasing the squeezing level by spatial filtering is the increased phase sensitivity shown in Fig. 3. For this reason it is not possible to decrease the pinhole radius indefinitely to increase squeezing. However, the phase width of the wider pinhole in Fig. 3 is still acceptably large at $\delta=3.8 \times 10^{-3}$. It may be possible to find an optimal pinhole radius for a given phase jitter in an experimental setting.

In the weak-focusing regime, the required nonlinear drive and the optimal phase difference are not much influenced by changes in the pinhole radius, whereas squeezing increases dramatically with increased pinhole radius. Therefore spatial filtering is useful in this regime as well.

In the no-diffraction limit $\left(l / z_{0} \rightarrow 0\right)$ and from Eqs. (22)-(24), the Fano factor is found to be 


$$
F=\frac{\exp \left[-4 \Phi \exp \left(-R_{\mathrm{ph}}{ }^{2}\right)\right]-\exp (-4 \Phi)}{2\left\{\exp \left[-2 \Phi \exp \left(-R_{\mathrm{ph}}{ }^{2}\right)-\exp (-2 \Phi)\right\}\right.} .
$$

Equation (37) reveals that the 3 - $\mathrm{dB}$ limit for the case with no pinhole $\left(R_{\mathrm{ph}} \rightarrow \infty\right)$ disappears when a pinhole is used.

\section{CONCLUSIONS}

In conclusion, we have shown that amplitude squeezing in excess of $4 \mathrm{~dB}$ can be generated in a practical experiment based on a Gaussian-beam-pumped degenerate optical parameter amplifier. In such an experiment it is critical to adjust the focused spot size of the pump beam such that the crystal length-to-confocal parameter ratio is close to 1.6. It is also crucial to adjust the pump power to reach the maximum possible squeezing level. The maximum required pump power and crystal length, however, are much lower than they would have to be in a comparable weak-focusing setup. Furthermore, working in the tight-focusing regime requires much less phase stability between the pump and the input signal beams. Aside from these differences, the DOPA crystal should preferably be noncritically phase matched and have a large acceptance angle. When these requirements are satisfied, a Gaussian-beam DOPA is an attractive device for generating high-power broadband amplitude-squeezed light at room temperature. When the DOPA is combined with linear spatial filtering with a simple pinhole at the output, relatively high levels of amplitude squeezing are possible.

O. Aytür's e-mail address is aytur@ee.bilkent.edu.tr.

\section{REFERENCES}

1. L. Mandel and E. Wolf, Optical Coherence and Quantum Optics (Cambridge U. Press, Cambridge, 1995), Chap. 12.

2. R. Short and L. Mandel, "Observation of sub-Poissonian photon statistics," Phys. Rev. Lett. 51, 384-387 (1983).

3. M. C. Teich and B. E. A. Saleh, "Observation of subPoissonian Franck-Hertz light at 253.7 nm," J. Opt. Soc. Am. B 2, 275-282 (1985).

4. S. Machida and Y. Yamamoto, "Observation of subPoissonian photoelectron statistics in a negative feedback semiconductor laser," Opt. Commun. 57, 290-296 (1986).

5. S. Machida and Y. Yamamoto, "Ultrabroadband amplitude squeezing in a semiconductor laser," Phys. Rev. Lett. 60, 792-794 (1988).

6. S. Machida and Y. Yamamoto, "Observation of amplitude squeezing from semiconductor lasers by balanced direct detectors with a delay line," Opt. Lett. 14, 1045-1047 (1989).

7. W. H. Richardson and R. M. Shelby, "Nonclassical light from a semiconductor laser operating at $4 \mathrm{~K}$," Phys. Rev. Lett. 64, 400-403 (1990).

8. M. J. Freeman, H. Wang, D. G. Steel, R. Craig, and D. R. Scifres, "Amplitude-squeezed light from quantum-well lasers," Opt. Lett. 18, 379-381 (1993).

9. H. Wang, M. J. Freeman, and D. G. Steel, "Squeezed light from injection-locked quantum well lasers," Phys. Rev. Lett. 71, 3951-3954 (1993).

10. W. H. Richardson, S. Machida, and Y. Yamamoto, "Squeezed photon-number noise and sub-Poissonian electrical partition noise in a semiconductor laser," Phys. Rev. Lett. 66, 2867-2870 (1991).

11. S. Machida, Y. Yamamoto, and Y. Itaya, "Observation of amplitude squeezing in a constant-current-driven semiconductor laser," Phys. Rev. Lett. 58, 1000-1003 (1987).

12. M. J. Freeman, H. Wang, D. G. Steel, R. Craig, and D. R. Scifres, "Wavelength-tunable amplitude-squeezed light from a room-temperature quantum-well laser," Opt. Lett. 18, 2141-2143 (1993).

13. M. J. Freeman, D. C. Kilper, D. G. Steel, R. Craig, and D. R. Scifres, "Room-temperature amplitude-squeezed light from an injection-locked quantum-well laser with a time-varying drive current," Opt. Lett. 20, 183-185 (1995).

14. J. Kitching, D. Provenzano, and A. Yariv, "Generation of amplitude-squeezed light from a room-temperature FabryPerot semiconductor laser," Opt. Lett. 20, 2526-2528 (1995).

15. J. Kitching and A. Yariv, "Room temperature generation of amplitude squeezed light from a semiconductor laser with weak optical feedback," Phys. Rev. Lett. 74, 3372-3375 (1995)

16. P. R. Tapster, J. G. Rarity, and J. S. Satchell, "Generation of sub-Poissonian light by high-efficiency light-emitting diodes," Europhys. Lett. 4, 293-299 (1987).

17. P. R. Tapster, J. G. Rarity, and J. S. Satchell, "Use of parametric down-conversion to generate sub-Poissonian light," Phys. Rev. A 37, 2963-2967 (1988).

18. J. Mertz, A. Heidmann, C. Fabre, E. Giacobino, and S. Reynaud, "Observation of high-intensity sub-Poissonian light using an optical parametric oscillator," Phys. Rev. Lett. 64, 2897-2900 (1990)

19. S. F. Pereira, M. Xiao, H. J. Kimble, and J. L. Hall, "Generation of squeezed light by intracavity frequency doubling," Phys. Rev. A 38, 4931-4934 (1988).

20. R. Kürz, R. Paschotta, K. Fiedler, and J. Mlynek, "Bright squeezed light by second-harmonic generation in a monolithic resonator," Europhys. Lett. 24, 449-454 (1993).

21. A. Sizmann, R. J. Horowicz, G. Wagner, and G. Leuchs, "Observation of amplitude squeezing of the up-converted mode in second harmonic generation," Opt. Commun. 80, 138-142 (1990).

22. R. Paschotta, M. Collett, P. Kürz, K. Fiedler, H. A. Bachor and J. Mlynek, "Bright squeezed light from a singly resonant frequency doubler," Phys. Rev. Lett. 72, 3807-3810 (1994).

23. H. Tsuchida, "Generation of amplitude-squeezed light at $431 \mathrm{~nm}$ from a singly resonant frequency doubler," Opt. Lett. 20, 2240-2242 (1995).

24. D. K. Serkland, P. Kumar, M. A. Arbore, and M. M. Fejer, "Amplitude squeezing by means of quasi-phase-matched second-harmonic generation in a lithium niobate waveguide," Opt. Lett. 22, 1497-1499 (1997).

25. R. D. Li, S. K. Choi, C. Kim, and P. Kumar, "Generation of sub-Poissonian pulses of light,” Phys. Rev. A 51, 3429-3432 (1995).

26. E. M. Daly, A. S. Bell, E. Riis, and A. I. Ferguson, "Generation of picosecond squeezed pulses using an all-solid-state cw mode-locked source," Phys. Rev. A 57, 3127-3130 (1998).

27. R. D. Li, S. K. Choi, and P. Kumar, "Gaussian-wave theory of sub-Poissonian light generation by means of travellingwave parametric deamplification," Quantum Semiclassic. Opt. 7, 705-713 (1995).

28. S. H. Youn, S. K. Choi, P. Kumar, and R. D. Li, "Observation of sub-Poissonian light in traveling-wave secondharmonic generation," Opt. Lett. 21, 1597-1599 (1996).

29. K. Schneider, R. Bruckmeier, H. Hansen, S. Schiller, and J. Mlynek, "Bright squeezed-light generation by a continuouswave semimonolithic parametric amplifier," Opt. Lett. 21, 1396-1398 (1996)

30. D. Levandovsky, M. Vasilyev, and P. Kumar, "Amplitude squeezing of light by means of a phase-sensitive fiber parametric amplifier," Opt. Lett. 24, 984-986 (1999).

31. Y. Yamamoto, S. Machida, and O. Nilsson, "Amplitude squeezing in a pump-noise-suppressed laser oscillator," Phys. Rev. A 34, 4025-4042 (1986).

32. C. Kim and P. Kumar, "Tunable sub-Poissonian light 
generation from a parametric amplifier using an intensity feedforward scheme," Phys. Rev. A 45, 5237-5242 (1992).

33. R. D. Li and P. Kumar, "Quantum-noise reduction in traveling-wave second-harmonic generation," Phys. Rev. A 49, 2157-2166 (1994).

34. K. G. Köprülü and O. Aytür, "Analysis of Gaussian-beam degenerate optical parametric amplifiers for the generation of quadrature-squeezed states," Phys. Rev. A 60, 41224134 (1999)

35. C. M. Caves, "Particles and fields," Phys. Rev. D 26, 18171839 (1982).

36. A. La Porta and R. Slusher, "Squeezing limits at high parametric gains," Phys. Rev. A 44, 2013-2022 (1991). 